See discussions, stats, and author profiles for this publication at: https://www.researchgate.net/publication/3975109

\title{
Space time coding schemes for 4 or more antennas
}

Conference Paper · October 2002

DOI: 10.1109/PIMRC.2002.1046665 · Source: IEEE XPlore

2 authors:

Mario Marques da Silva

Universidade Autónoma de Lisboa Luís de Camoes

63 PUBLICATIONS 227 CITATIONS

SEE PROFILE

Some of the authors of this publication are also working on these related projects:

FRAMES View project
A. Correia

ISCTE-Instituto Universitário de Lisboa

124 PUBLICATIONS 526 CITATIONS

SEE PROFILE 


\title{
SPACE TIME CODING SCHEMES FOR 4 OR MORE ANTENNAS
}

\author{
Mário Marques da Silva, Américo Correia \\ Instituto de Telecomunicacões, ADETTI \\ IST, Torre Norte 11.10, Av. Rovisco Pais, 1049-001 Lisboa, Portugal \\ marquesilva@mail.telepac.pt, americo.correia@lx.it.pt
}

\begin{abstract}
In this paper we present a new space time transmit diversity (STTD) scheme for $M \geq 4$ transmit antennas and one receive antenna. The new diversity scheme belongs to the open loop class and consists of an extension to the work presented in $[1,2,3]$. Another hybrid scheme that combines open and close loop classes is also presented. The coding rate of the new scheme is one and in spite of the presence of inter-symbol interference it may achieve the full diversity after applying a multi-dimensional orthonormal rotation to the original constellation. For each coding scheme every block with 8 received symbols is detected using a maximum likelihood decoding algorithm to get the estimated maximum likelihood sequence. Q-QAM modulations are analysed.
\end{abstract}

Keywords - Rayleigh fading, antenna transmission diversity, multi-dimensional constellation and rotation, inter-symbol interference.

\section{INTRODUCTION}

To achieve a high spectral efficiency, i.e., a coding rate equal to one, space time transmit diversity coding schemes are based on the transmission of different information symbols over the different time slots and antennas.

The new transmission diversity scheme is spectral efficient and resistant to fading, where the base station uses $M=4$ transmitting antennas and the mobile only has one receiving antenna. As long as the antennas are spaced sufficiently far apart, the transmission from each antenna undergoes independent fading.

In [1] Alamouti has presented a new diversity scheme known as Space Time Transmit Diversity (STTD), later on accepted as an option on the 3GPP Specifications for the downlink of the Wide-band CDMA (WCDMA) system.

The new diversity scheme belongs to the open loop class, where feed-forward or training information, is sent in order to have knowledge of the channel at the transmitter. There is $M \geq 4$ transmitting antennas instead of only two. Tarokh in [2] has shown that there are no orthogonal complex designs for $M=4$, with coding rate one. As the new diversity scheme has coding rate one, it considers a non-orthogonal complex design, where inter-symbol interference can not be avoided. The full diversity is only achieved after applying a multidimensional orthonormal rotation to the original constellation. This scheme is applied on WCDMA where extra inter-symbol interference is avoided due to the use of wide-band signals.

\section{SYSTEM MODELS}

The system model to be studied is the downlink of a WCDMA (Wide-band Code Division Multiple Access) system where there are $M \geq 4$ transmitter antennas (base station) and a single receiver antenna (mobile). The communication links between the four transmitting antennas and the receiving one are not generally line-of-sight. The multipath Rayleigh fading model is generally assumed. Between the receiver antenna and each transmitting antenna there are $K$ multipath, where each one has equal average energy. The probability density function for each link is given by [4]:

$$
f\left(\gamma_{i}\right)=\frac{\gamma_{i}^{K-1}}{(K-1) ! \Gamma^{K}} \exp \left(-\gamma_{i} / \Gamma\right), \quad \gamma_{i}>0
$$

$\Gamma$ is the average signal to noise ratio of each path. The assumption of independent fading is valid if the transmitter antennas or time slots are spaced sufficiently far apart.

We assume wideband signals and Rake receivers that are able to estimate accurately the fading of the $k$-th multipath of the $i$-th transmitting antenna, $h_{i, k} . h_{i, k}$ represents a circular complex Gaussian random variable with zero mean and variance 0.5 per dimension. The matrix $S_{12}$ with the transmitted symbols will be introduced for further use [1]

$$
S_{12}=\left[\begin{array}{cc}
s_{1} & S_{2} \\
-s_{2}^{*} & S_{1}^{*}
\end{array}\right]
$$


* means complex conjugate. The total power transmitted on $M$ antennas at one symbol time is constant and independent of $M$. The new scheme has $M=4$ transmitting antennas (see Figure 1). The symbol construction includes the matrix (2) and can be written as [3]

$$
S_{14}=\left[\begin{array}{cc}
S_{12} & S_{34} \\
S_{34}^{*} & -S_{12}^{*}
\end{array}\right]
$$

The received symbols in four consecutive symbol intervals are respectively given by:

$$
\begin{aligned}
& r_{1}=h_{1} s_{1}-h_{2} s_{2}^{*}+h_{3} s_{3}^{*}-h_{4} s_{4}+n_{1} \\
& r_{2}=h_{1} s_{2}+h_{2} s_{1}^{*}+h_{3} s_{4}^{*}+h_{4} s_{3}+n_{2} \\
& r_{3}=h_{1} s_{3}-h_{2} s_{4}^{*}-h_{3} s_{1}^{*}+h_{4} s_{2}+n_{3} \\
& r_{4}=h_{1} s_{4}+h_{2} s_{3}^{*}-h_{3} s_{2}^{*}-h_{4} s_{1}+n_{4}
\end{aligned}
$$

$r_{l}$ is the symbol arrived in the first symbol interval and $r_{2}$ is the symbol arrived in the second interval, and so on. $h_{1} h_{2} h_{3}$ $h_{4}$ are complex random variables, characteristic of the single path fading channel, where each amplitude has a Rayleigh distribution and the phase is uniformly distributed in $[0,2 \pi]$, $n_{1}$ up to $n_{4}$ are gaussian random variables, with variance $\mathrm{No} / 2$. Note that the mobile receiver only has a single antenna.

The receiver to demodulate the signals does the following processing:

$$
\begin{aligned}
& z_{1}=r_{1} h_{1}^{*}+r_{2}^{*} h_{2}-r_{3}^{*} h_{3}-r_{4} h_{4}^{*}=\sum_{i}\left|h_{i}\right|^{2} s_{1}-c_{0} s_{4}+\eta_{1} \\
& z_{2}=r_{2} h_{1}^{*}-r_{1}^{*} h_{2}-r_{4}^{*} h_{3}+r_{3} h_{4}^{*}=\sum_{i}\left|h_{i}\right|^{2} s_{2}+c_{0} s_{3}+\eta_{2} \\
& z_{3}=r_{3} h_{1}^{*}+r_{4}^{*} h_{2}+r_{1}^{*} h_{3}+r_{2} h_{4}^{*}=\sum_{i}\left|h_{i}\right|^{2} s_{3}+c_{0} s_{2}+\eta_{3} \\
& z_{4}=r_{4} h_{1}^{*}-r_{3}^{*} h_{2}+r_{2}^{*} h_{3}-r_{1} h_{4}^{*}=\sum_{i}\left|h_{i}\right|^{2} s_{4}-c_{0} s_{1}+\eta_{4}
\end{aligned}
$$

$c_{0}=2 \cdot \operatorname{Re}\left\{h_{1}^{*} h_{4}-h_{2} h_{3}^{*}\right\}$

We conclude from (5) that there is inter-symbol interference (IIS) from only one symbol in the decoding of any other symbol. This means that the full diversity of order $4 K$ is not directly achievable. We need to compensate for the IIS in order to achieve the full diversity. However, as we keep the coding rate as one, we are not allowed to repeat the transmission of any symbol. This means that maximum likelihood sequence estimation alone is not effective. We need to first group (and later on transmit) $L=2^{l}$ ( 1 is a positive integer greater than two) consecutive symbols and multi-dimensional rotate to a new constellation $[5,6]$. Instead of transmitting QPSK, or 16QAM super-symbols, represented by vectors in constellation $\mathrm{X}$, every $L \geq 8$ symbols are grouped and multi-dimensionally rotated, i.e, they are multiplied by an orthonormal complex rotation matrix $\mathrm{A}_{8}$,

$$
A_{4}=\left[\begin{array}{cccc}
e^{j \varphi} & -j e^{j \varphi} & e^{j \varphi} & -j e^{j \varphi} \\
-j e^{-j \varphi} & e^{-j \varphi} & -j e^{-j \varphi} & e^{-j \varphi} \\
e^{-j \varphi} & -j e^{-j \varphi} & -e^{-j \varphi} & j e^{-j \varphi} \\
j e^{j \varphi} & e^{j \varphi} & -j e^{j \varphi} & -e^{j \varphi}
\end{array}\right]
$$

$A_{8}=\left[\begin{array}{cc}A_{4} & A_{4} \\ A_{4}^{*} & -A_{4}^{*}\end{array}\right] / 2 \sqrt{2}$

$\varphi$ is the angle chosen to minimize the BER performance. The rotated symbols are located in a new constellation, $S=$ $A_{8} X$. So the rotated symbols $\mathrm{s}_{1} \mathrm{~s}_{2} \mathrm{~s}_{3} \mathrm{~s}_{4}$ and $\mathrm{s}_{5} \mathrm{~s}_{6} \mathrm{~s}_{7} \mathrm{~s}_{8}$ are sent to antenna 1 in two consecutive periods each one with duration of four symbols $4 T$ (see Figure 1). 8 symbols are detected every $8 T$.

With or without multi-dimensional rotation each supersymbol includes $L=8$ elements and the maximum likelihood receiver must choose between $q^{L}$ possible combinations of $(\mathrm{S}, \mathbb{S})$.

$$
\operatorname{Min}\left[\left.\sum_{j}\left|z_{j}-\sum_{i}\right| h_{i}\right|^{2} s_{i} \pm\left. c_{0} s_{k}\right|^{2}\right] \forall(\mathrm{S}, S) .
$$

$k \neq j, 1 \leq i, j \leq M$. there are $q^{L}$ different combinations of $(\mathbf{S}, \mathbb{S})$. $q$ is the alphabet size, i.e. $q=4$ or 16 for QPSK or 16QAM, respectively. For increasing values of $q$ and $L$ it is suggested the use of optimal detection like the Lattice decoder of [7]. An alternative transmit diversity scheme with 4 antennas is presented in Figure 2. This is a hybrid scheme that employs the Alamouti scheme (open loop class) with feedback indication (FBI) about the state of the Rayleigh fading channel (close loop class). Within this scheme only the two 'best' antennas transmit information and signaling data while the other two antennas only transmit signaling data at a lower bit rate (in our case we assume an overhead of $10 \%)$. 
$8 \mathrm{~T} \quad 7 \mathrm{~T} \quad 6 \mathrm{~T} \quad 5 \mathrm{~T} \quad 4 \mathrm{~T} \quad 3 \mathrm{~T} \quad 2 \mathrm{~T} \quad \mathrm{~T} \quad 0$

\begin{tabular}{cccccccccc}
\hline $\mathrm{S}_{8}$ & $\mathrm{~S}_{7}$ & $\mathrm{~S}_{6}$ & $\mathrm{~S}_{5}$ & $\mathrm{~S}_{4}$ & $\mathrm{~S}_{3}$ & $\mathrm{~S}_{2}$ & $\mathrm{~S}_{1}$ & Antenna 1 \\
\hline $\mathrm{S}_{7}^{*}$ & $-\mathrm{S}_{8}^{*}$ & $\mathrm{~S}_{5}^{*}$ & $-\mathrm{S}_{6}^{*}$ & $\mathrm{~S}_{3}^{*}$ & $-\mathrm{S}_{4}^{*}$ & $\mathrm{~S}_{1}^{*}$ & $-\mathrm{S}_{2}^{*}$ & Antenna 2 \\
\hline$-\mathrm{S}_{6}^{*}$ & $-\mathrm{S}_{5}^{*}$ & $\mathrm{~S}_{8}^{*}$ & $\mathrm{~S}_{7}^{*}$ & $-\mathrm{S}_{2}^{*}$ & $-\mathrm{S}_{1}^{*}$ & $\mathrm{~S}_{4}^{*}$ & $\mathrm{~S}_{3}^{*}$ & \\
\hline$-\mathrm{S}_{5}$ & $\mathrm{~S}_{6}$ & $\mathrm{~S}_{7}$ & $-\mathrm{S}_{8}$ & $-\mathrm{S}_{1}$ & $\mathrm{~S}_{2}$ & $\mathrm{~S}_{3}$ & $-\mathrm{S}_{4}$ & Antenna 4
\end{tabular}

Figure 1: Transmission diversity scheme (STBC)

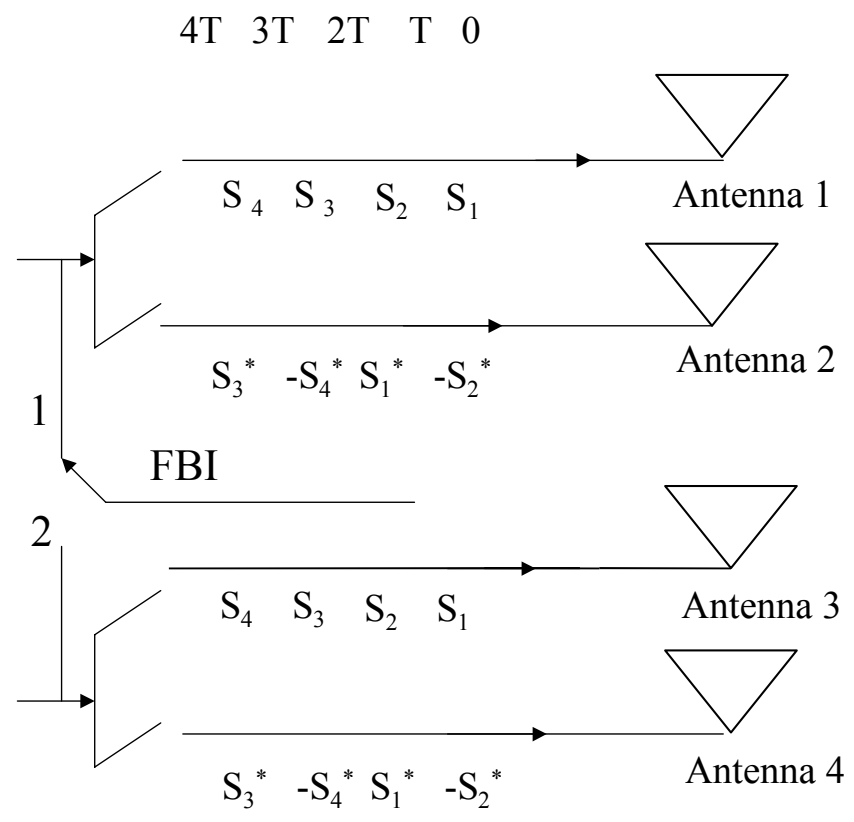

Figure 2: Transmission diversity scheme (STTD2+STD)

\section{NUMERICAL RESULTS AND DISCUSSION}

Regarding the simulations the Monte Carlo approach is considered, i.e., drawing a random (independent) sequence of channel parameters, in accordance with the distribution of these parameters. For each set of drawn parameters the BER (bit error rate) is evaluated and after a sufficiently large number of parameter sets (in our case $2^{17}$ ) the estimate of the BER distribution is constructed (we restrict ourselves to the signal to noise ratio interval $\mathrm{Es} / \mathrm{No}=[0,12 \mathrm{~dB}]$ ).

Note that the transmission diversity scheme here denoted as complex rotation matrix (CRM), is independent of the number of antennas $M$. Stand alone, this scheme includes only the initial signal processing of the new scheme. $L=8$ symbols are grouped and multi-dimensionally rotated, i.e, they are first multiplied by one orthonormal complex rotation matrix (CRM), later on they are time interleaved to ensure independent fading and transmitted through a single antenna.

We will combine the CRM scheme with the space-time block code (STBC) with coding rate one to achieve a higher transmission diversity. The combined scheme here studied will be denoted in the next figures as CRM+STBC.

For all diversity schemes (including the Alamouti scheme with $M=2$ antennas, denoted as STTD2), the detection considers always sequences with length $L=8$, to get the same decoding complexity. The Alamouti scheme was designed to work with two antennas and there is no extension for four antennas, as was shown in [2]. For STBC, the several schemes considered always four transmitting antennas [3] and one receiving antenna. In the results, STBC2 means four transmitting antennas and single-path faded channel, while STBC4 means four antennas and twopath faded channel. Additionally, STTD2 means the Alamouti scheme with two transmitting antennas for a single-path faded channel, while STTD4 means the maximum achievable diversity with four antennas for a single-path faded channel, but it is never achieved since this is an ideal scheme that does not exist in reality [3]. Similarly, STTD8 means an ideal scheme for a system with eight antennas over a single-path Rayleigh faded channel.

Figure 3 presents the BER performance of several transmission diversity schemes where the reference modulation considered was QPSK (4-QAM). We observe that for $\mathrm{BER}=10^{-2}$, the additional diversity gain provided by the ideal scheme with $M=4$ antennas (denoted as STTD4) is $2.0 \mathrm{~dB}$ compared to STTD with $M=2$ antennas (STTD2). The real scheme has also 4 antennas (denoted as STBC) and achieves $1.6 \mathrm{~dB}$ of additional diversity gain, i.e. a loss of $0.5 \mathrm{~dB}$ compared to the ideal scheme (loss due to intersymbol interference (IIS)). For $\mathrm{BER}=2 \times 10^{-3}$ the loss increases to $1 \mathrm{~dB}$ approximately. The new scheme $\mathrm{CRM}+\mathrm{STBC}$, for $\mathrm{BER}=10^{-3}$ offers the gain of $0.8 \mathrm{~dB}$ approximately compared to the ideal scheme with $M=4$.

Figure 4 presents the BER performance of several transmission diversity schemes, for several Rayleigh faded 
channels and the 16-QAM modulation. We observe that for $\mathrm{BER}=10^{-2}$, the additional diversity gain provided by the ideal scheme with $M=4$ antennas (STTD4) is $1.7 \mathrm{~dB}$ compared to STTD2. The real scheme achieves $1.2 \mathrm{~dB}$ of additional diversity gain, i.e., a loss of $0.5 \mathrm{~dB}$ compared to the ideal scheme. For the Rayleigh fading channel with 2 paths due to the additional IIS generated the loss increases to $1.2 \mathrm{~dB}$ for $\mathrm{BER}=2 \times 10^{-3}$ between the real $(\mathrm{STBC} 4)$ and the ideal (STTD8) schemes. Due to the excessive computing time of the ML algorithm, there are not available results for the scheme CRM+STBC with 16-QAM.

In figure 5 the modulation considered is also QPSK. The BER performance of the transmission diversity scheme (STTD2+STD) is presented and compared with the single path (STBC2 vs STTD2) and two-paths (STBC4) Rayleigh faded channels. The most important observation is the same BER performance of the diversity scheme CRM+STBC (see figure 3) compared to the STTD2+STD. As long as a lattice decoder is used the complexity of the association CRM with STBC is lower than the complexity of the scheme STTD2+STD. The scheme STBC does not work properly with selective transmission diversity (STD), because it is unable to compensate for the additional IIS, in spite of providing a higher diversity order compared to the scheme without STD.

Figure 6 corresponds to figure 5 with the 16-QAM modulation. All the conclusions drawn for the previous modulation are valid for the 16-QAM. The only difference is an expected increase of the BER

\section{CONCLUSIONS}

The new transmission diversity scheme here presented is a space-time block coding (STBC) for complex signals with coding rate one that employs $M=4$ transmit antennas. In spite of the unavoidable inter-symbol interference it achieves the full diversity when combined with a multidimensional complex rotation matrix (CRM) that rotates the original constellation. As long as the number of symbols involved in the multi-dimensional rotation matrix $L$ is greater than $M(L=8, M=4)$, the proposed new diversity scheme $\mathrm{CRM}+\mathrm{STBC}$ is able to compensate for the diversity loss due to inter-symbol interference.

Another way to increase the transmission diversity order keeping constant $M=4$, is obtained combining the Alamouti scheme with 2 antennas and a selective transmit diversity (STD) scheme where only the two best antennas transmit the information data.

\section{REFERENCES}

[1] Alamouti, S. M., "A Simple Transmitter Diversity Scheme for Wireless Communications”, IEEE JSAC, Oct. 1998, p. 1451-1458.

[2] Tarokh et al.,'Space-time block codes from orthogonal designs", IEEE Trans. Inform. Theory, pp. 1456-1467, July 1999.

[3] Tarokh et. Al. .,'Space-time codes for High Data Rate Wireless Communication: Performance criterion and Code Construction", IEEE Trans. Inform. Theory, pp. 744-765, Mar. 1998.

[4] Proakis J. G. "Digital Communications", McGraw Hill 1995

[5] Silva. M, Correia A. "Space Time Diversity for the Downlink of WCDMA", Proc. WPMC'2001, Aalborg, Denmark, Sep. 2001.

[6] Correia A. "Complex Constellations for Transmission Diversity", to be published at Wireless Personal Communications Journal of Kluwer Academic Pub..

[7] Damen O. et al., "Lattice Code Decoder for Space-Time Codes",IEEE.Communicatons Letters, Vol. 4. No. 5, May 2000, p. 161-163. 


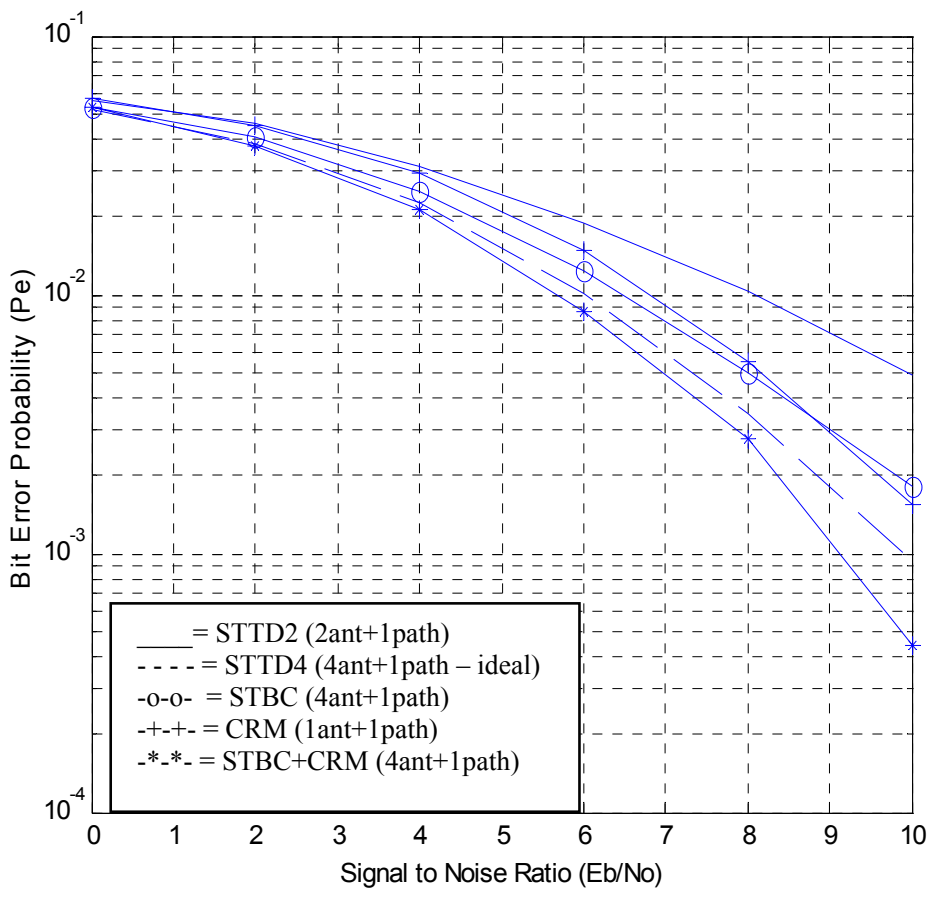

Figure 3: BER, 4-QAM (Reference scheme STBC)

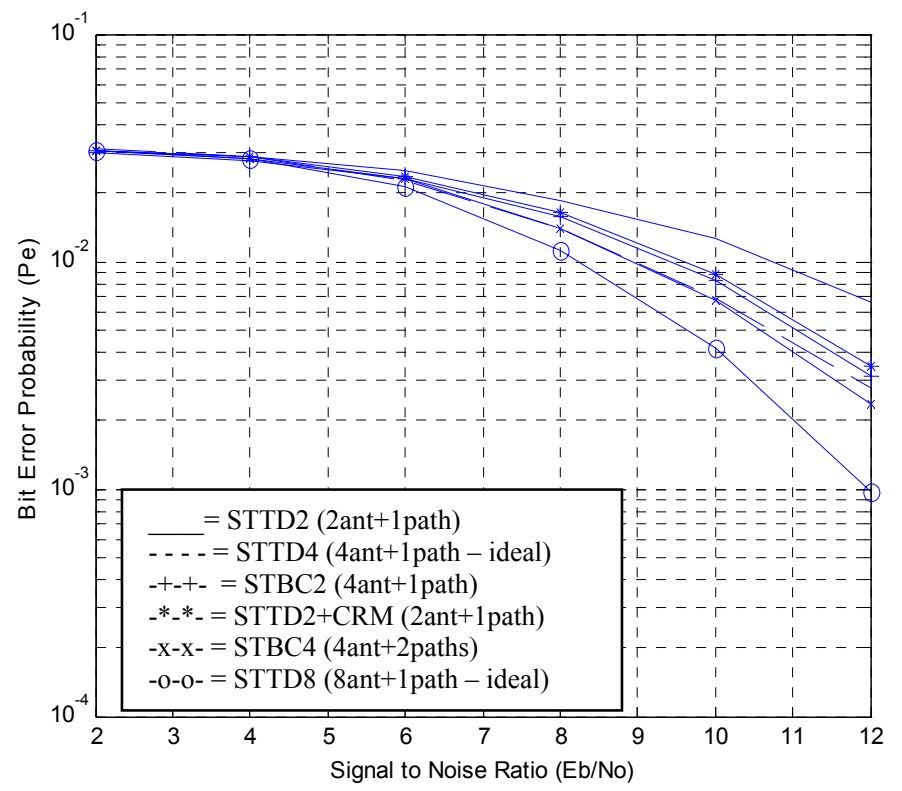

Figure 4: BER, 16-QAM (Reference scheme STBC)

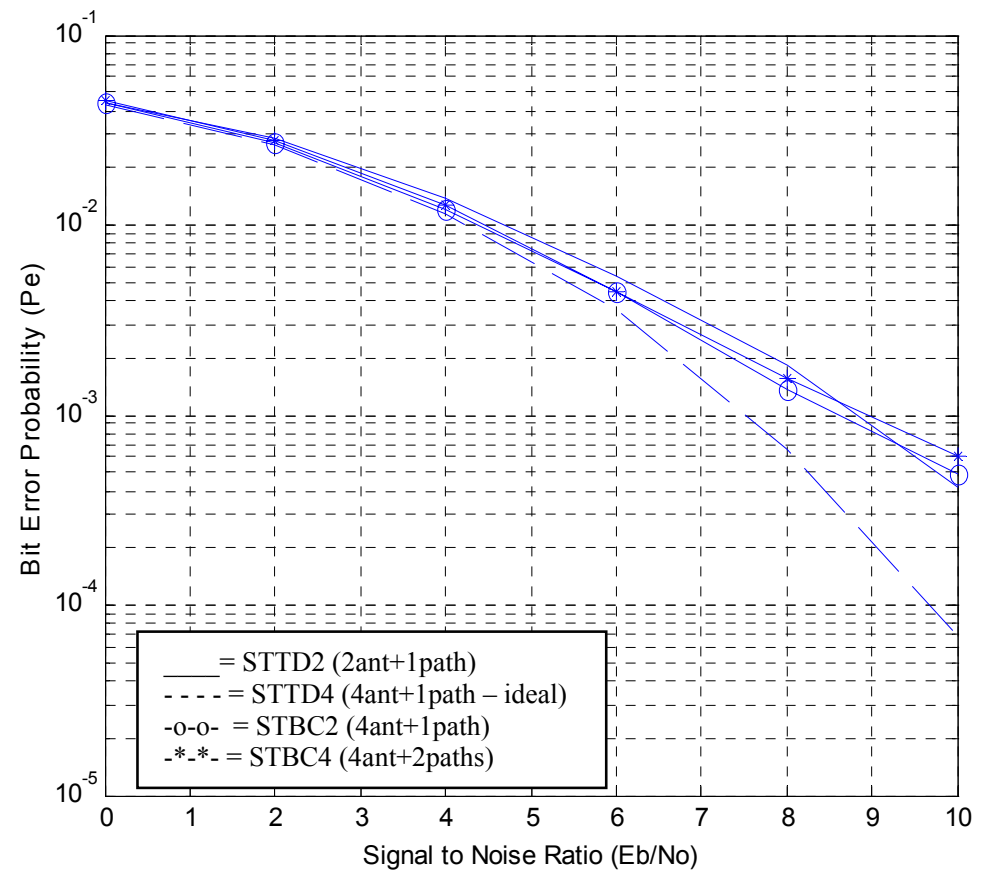

Figure 5: BER, 4-QAM (Reference scheme STTD2STD)

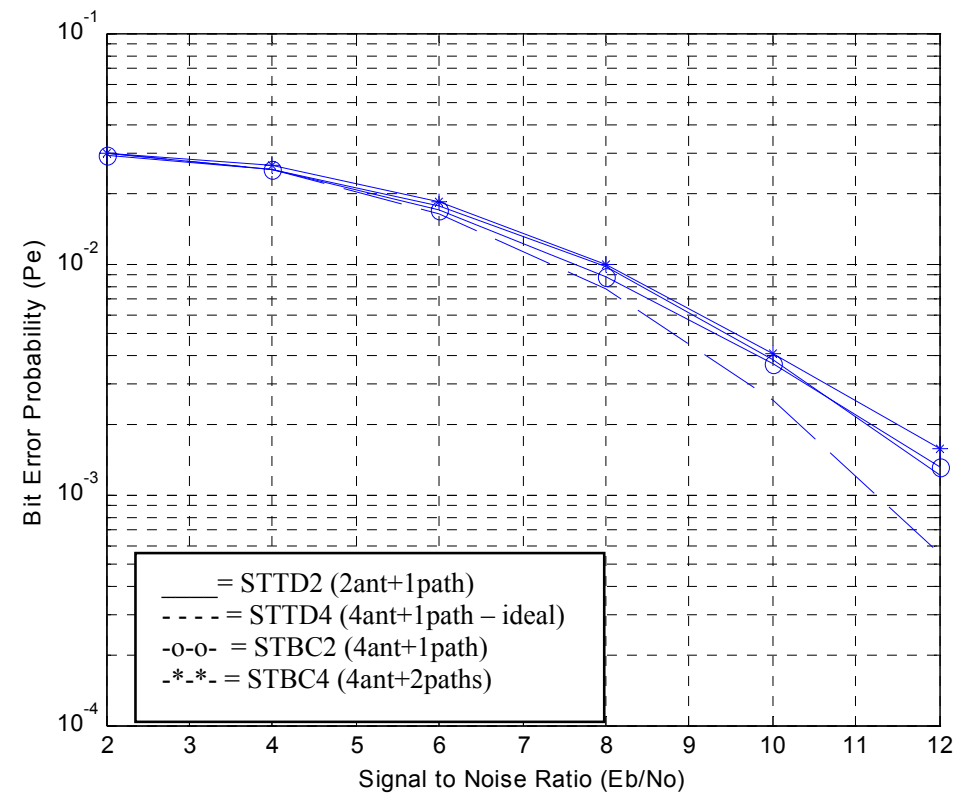

Figure 6: BER, 16-QAM (Reference scheme STTD2STD) 